\section{In the news}

\section{STAYING POWER}

The largest study to evaluate the oral therapy imatinib (Glivec/STI571) in patients with chronic myeloid leukaemia $(\mathrm{CML})$ reported a survival rate of nearly $90 \%$ after 5 years of treatment.

Five-year follow-up data from the International Randomized Interferon versus STI571 (IRIS) trial were presented at the American Society of Clinical Oncology annual meeting. IRIS enrolled 1,106 newly diagnosed Philadelphia-chromosome-positive CML patients. Of the 553 patients randomized to imatinib rather than standard therapy, $69 \%$ remain on imatinib and, of those, overall survival is $89 \%$ and disease-specific survival is $95 \%$ after 5 years.

"This trend, if it holds, coupled with the low risk of relapse, means that the possibility of long-term survival with CML is increasingly likely," said Brian Druker, the lead author of the study at Oregon Health and Science University (http://www. reuters.com, 3 June 2006).

Furthermore, data from the study also showed that the percentage of patients with a complete cytogenetic response actually increased from $69 \%$ to $87 \%$ between the first and fifth years of treatment. "Very few oncology medicines offer patients the opportunity to achieve better outcomes the longer they take the therapy," said David Epstein, of Novartis Oncology (http://www. novartis.com, 3 June 2006).

Although imatinib has been heralded as a 'life saver' by patients (http://news.bbc.co.uk, 6 June 2006), Druker, who helped develop imatinib, pointed out that even imatinib has its limits and is not a cure for CML. Over the 5 years of treatment in IRIS, approximately $18 \%$ of imatinibtreated patients experienced some form of disease progression, and $5 \%$ discontinued therapy because of adverse effects. "People have to remain on therapy and remain on it for the long term," said Druker (http://www.medpagetoday.com, 5 June 2006).

Sarah Seton-Rogers

\title{
TUMOUR METABOLISM
}

\section{Shifting the balance}

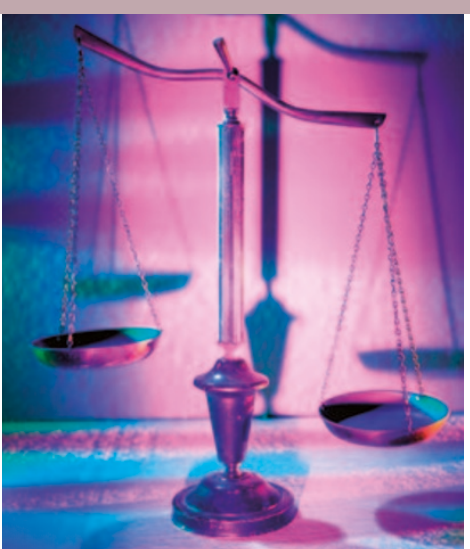

Upregulation of glycolysis, the anaerobic breakdown of glucose to produce ATP, occurs in almost every tumour. Paul Hwang and colleagues propose that such a widespread metabolic alteration must be stimulated by a pathway with similarly widespread alterations in cancer, and found that TP53, one of the most commonly mutated genes in human cancers, can alter the balance between glycolysis and aerobic respiration in tumour cells.

Cancer cells seem to increase glycolysis even in the presence of ample oxygen (a process termed the 'Warburg effect'), and glycolysis seems to correlate with tumour aggressiveness, indicating that it might be crucial in the evolution of malignancies. Hwang and colleagues set out to determine if loss of the tumour suppressor p53 could increase the level of glycolysis and reduce aerobic respiration.

The authors first showed that mitochondria prepared from mice with $\operatorname{Trp53}$ disruption showed significantly lower levels of oxygen consumption than Trp53 wild-type mice, and they observed a similar reduction in HCT116 human colon
4

TP53 ...

can alter

the balance

between

glycolysis

and aerobic

respiration in

tumour cells.

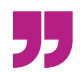

\section{DNA VACCINES}

\section{Provoking a response}

The inhibition of angiogenesis to reduce tumour progression has shown promise, but the short half-life and high production costs of endogenous angiogenesis inhibitors, such as angiostatin, have hindered clinical development. Lars Holmgren et al. now report that vaccination with DNA that encodes the angiostatin receptor, angiomotin, overcomes these problems and those of developing active immunotherapies by breaking immune tolerance and provoking an immune response against angiogenesis. In addition, the angiomotin vaccine inhibited tumour progression in mice when given in combination with a tumour-cell-targeted DNA vaccine.

The authors used cDNA that encodes the human $\mathrm{p} 80$ isoform of angiomotin inserted into a plasmid vector (pcDNA3Amot), and showed that transfection into the cervical cancer cell line, HeLa, led to expression of the angiomotin protein. Intramuscular injection and electroporation with pcDNA3-Amot into mice that were then challenged by the injection of mouse breast cancer cells led to the prolonged suppression of tumour growth compared with the injection of a control vector.

Next, the BALB-NeuT transgenic breast cancer mouse model (which is positive for rat epidermal-growth-factor receptor 2 (ERBB2, also known as HER2)) was used to investigate the ability of angiomotin to inhibit the angiogenic switch and tumour progression. The mice were vaccinated either before or after the onset of tumour angiogenesis, and although no effect on tumour progression was seen, vaccination reduced tumour angiogenesis by over $60 \%$. Importantly, pcDNA3-Amot vaccination did not negatively affect the vasculature of the surrounding stroma or the vessels 